\title{
Effect of protonation and hydrogen bonding on the fluorescent properties and exciplex formation of $N$-(4-pyridyl)-1,2- naphthalimide
}

\author{
Krisztina Sebők-Nagy and László Biczók * \\ Chemical Research Centre, Hungarian Academy of Sciences, P.O. Box 17, 1525 Budapest, \\ Hungary
}

Received 9th December 2003, Accepted 17th December 2003

First published as an Advance Article on the web 20th January 2004

The effects of fluorinated hydroxy compounds and naphthalene on the fluorescence of $N$-(4-pyridyl)-1,2-

naphthalimide (PyNI) have been studied in toluene. The interaction of the pyridyl moiety of PyNI with hexafluoro2-propanol (HFIP) gave rise to a hydrogen-bonded complex, whereas a more stable, hydrogen-bonded ion pair was formed with trifluoroacetic acid (TFA). Time-resolved fluorescence measurements demonstrate that hydrogen bonding with HFIP is a reversible process, even in the excited state, and revealed the rate constants of the various energy dissipation processes. The fluorescence yield enhancement of about one order of magnitude upon the $1: 1$ binding of PyNI to HFIP or TFA is primarily attributed to the deceleration of the internal conversion, and the fluorescence proved to be the dominant deactivation pathway of the singlet excited complexes. Both PyNI and its TFA complex produced fluorescent exciplexes with naphthalene. Protonation of PyNI markedly decreases the energy of the exciplex, leading to faster radiationless energy dissipation as well as to slow dissociation into an excited PyNI-TFA complex and ground-state naphthalene.

\section{Introduction}

Strong organic acids exert a profound influence on the photochemical reactions and deactivation pathways of excited molecules. Photorearrangement, photoinduced substitution and dechlorination are promoted by the addition of trifluoroacetic acid (TFA). ${ }^{1}$ The fluorescence quenching and hydrogen exchange of simple aromatic compounds with TFA have also been studied extensively in order to unravel the kinetics and mechanisms of these reactions. ${ }^{2}$ Considerable acceleration of the photochemical electron transfer was found in the presence of acid when quinones served as electron acceptors. $^{3}$ Systematic investigation of 4-dimethylaminosubstituted meso-tetraphenylporphyrin derivatives revealed that charge-transfer excited state can be produced upon protonation. ${ }^{4}$

Photoinitiated processes of naphthalimides are of particular interest because of their widespread applications in biological and medical areas, as well as in supramolecular chemistry and materials science. The remarkable sensitivity of this type of chromophores to their microenvironment has been utilised in the development of fluorescent chemosensors. ${ }^{5}$ Polymers with naphthalimide pendant groups can generate patterned fluorescent images ${ }^{6}$ and the interplay between dopant, exciplex and host emission has been examined in organic light-emitting diodes. $^{7}$ The quenching ${ }^{8}$ and photoproduct formation ${ }^{9}$ of excited 1,8-naphthalimide derivatives with aromatic and unsaturated compounds were suggested to proceed via exciplexes. Although the photophysical characteristics ${ }^{10}$ and the photoreactions ${ }^{11}$ depend considerably on the position of the dicarboximide moiety on the naphthalene ring, the majority of the studies have dealt merely with the derivatives of the 1,8 -isomer.

We have recently shown that the introduction of the 4-pyridyl substituent onto the nitrogen of 1,2-naphthalimide markedly diminishes the fluorescence quantum yield, which is primarily due to acceleration of the internal conversion, and hydrogen bonding with phenols can lead to static quenching via electron transfer. ${ }^{12}$ As an extension of this investigation, we now reveal how the addition of trifluoroacetic acid influences the fluorescent behaviour and the kinetics of the excited-state deactivation pathways of $N$-(4-pyridyl)-1,2-naphthalimide (PyNI). To distinguish the changes caused by protonation from those originating from hydrogen bonding, the effect of weakly acidic 1,1,1,3,3,3-hexafluoro-2-propanol is also examined. In addition, we unravel how the protonation and the hydrogen bonding of PyNI affect the kinetics of exciplex formation with naphthalene. Studies have been carried out in toluene because the strongest interaction with additives is expected in non-polar media. The structures of the investigated compounds are shown in Scheme 1.

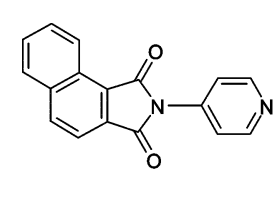

PyNI

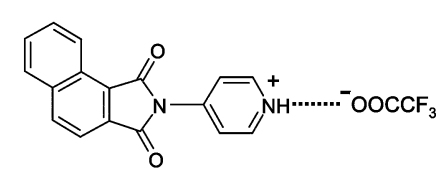

PyNIH $^{+}$<smiles></smiles>

PyNIH

Scheme 1

\section{Experimental}

$N$-(4-Pyridyl)-1,2-naphthalimide was prepared by the condensation of 1,2-naphthalenedicarboxylic anhydride with 4-aminopyridine (Aldrich) in dimethylformamide. After recrystallisation from dimethylformamide, the product was purified by passing it through a silica gel column using $\mathrm{CHCl}_{3}$ as the eluent. Toluene (HPLC grade), trifluoroacetic acid and 1,1,1,3,3,3-hexafluoro2-propanol (HFIP) (Aldrich) were used as received. Naphthalene was recrystallised prior to use. UV-visible absorption spectra were obtained using a Hewlett-Packard 8452 diodearray spectrophotometer. Corrected fluorescence spectra were recorded on a Jobin-Yvon Fluoromax-P photon-counting spectrofluorometer. Fluorescence quantum yields were determined 
relative to that of 9,10-diphenylanthracene in cyclohexane, for which a reference yield of 0.90 was used. ${ }^{13}$ Fluorescence lifetimes were measured using the time-correlated singlephoton counting technique. Pulses from a Picoquant diode laser (pulse duration $60 \mathrm{ps}$ FWHM, wavelength $400 \mathrm{~nm}$ ) excited the samples and the emission was collected at a $90^{\circ}$ angle through an Oriel $0.25 \mathrm{~m}$ monochromator located in front of a Hamamatsu H5783 photosensor module. The output signal was sent to a Picoquant Timeharp 100 computer board module (channel width $36 \mathrm{ps}$ ). Data were analysed by a non-linear least-squares deconvolution method. Triplet yields were determined via the $\mathrm{XeCl}$ excimer laser flash photolysis technique, exploiting the efficient triplet sensitisation of perylene, as described in our previous paper. ${ }^{14}$ Cyclic voltammograms were recorded with a Cypress Systems OMNI-101 microprocessor-controlled potentiostat using a glassy carbon working electrode, a platinum counter electrode and a $\mathrm{Ag}-\mathrm{AgCl}$ reference electrode, with $0.1 \mathrm{M}$ tetrabutylammonium hexafluorophosphate as the supporting electrolyte in deoxygenated benzonitrile.

\section{Results and discussion}

\section{Protonation and hydrogen bonding in the ground and excited} states

The fluorescence behaviour of PyNI proved to be very sensitive to hydroxy compounds. To exclude quenching via electron transfer, additives with high oxidation potentials were used. Both TFA and HFIP are strong hydrogen-bond donors Abraham's $\log K_{a}$ parameters ${ }^{15}$ and the $\mathrm{p} K_{\mathrm{a}}$ values demonstrate that not only the acidity of TFA, but also its hydrogen-bonding ability, are significantly higher compared to HFIP. Values of 3.55 and 2.83 have been reported for $\log K_{a}$ of TFA and HFIP, respectively, and their $\mathrm{p} K_{\mathrm{a}}$ are 0.52 and 9.3 , respectively. ${ }^{15}$

Fig. 1 presents the characteristic changes in the absorption spectrum of PyNI with gradually increasing concentrations of the two additives. In the presence of HFIP, only a slight change in the spectrum is observed, which is indicative of hydrogen bonding. On the other hand, the much more pronounced

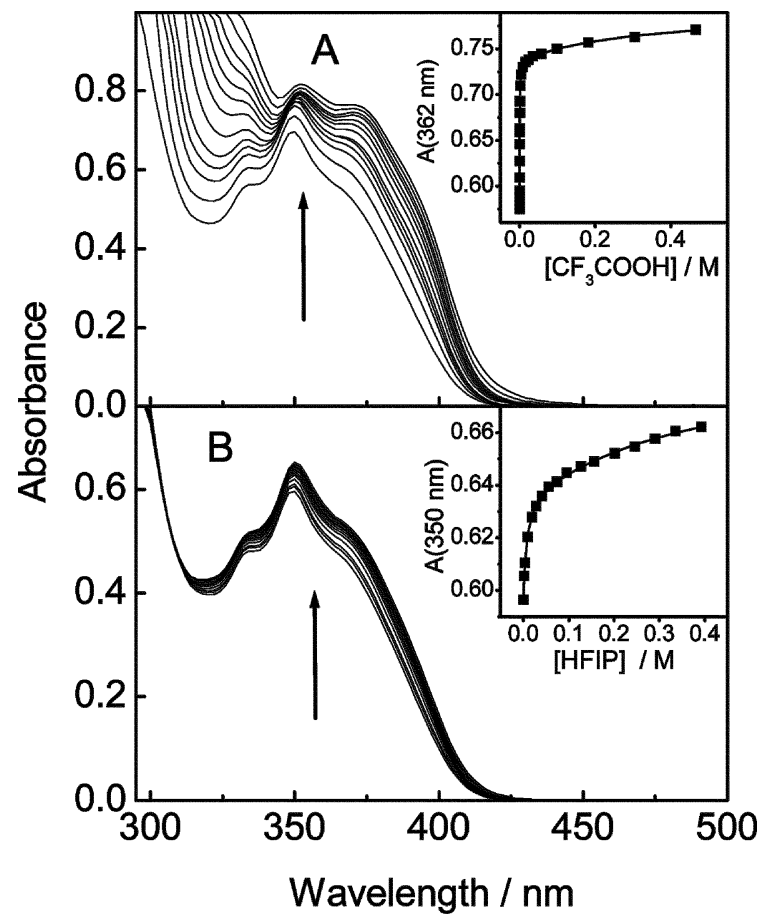

Fig. 1 Effect of TFA (A) and HFIP (B) on the absorption spectrum of PyNI in toluene. $[\mathrm{PyNI}]=0.328(\mathrm{~A})$ and $0.281 \mathrm{mM}(\mathrm{B})$; optical path 10 $\mathrm{mm}$. The insets show the rise in the absorbance with increasing additive concentration at 362 (A) and $350 \mathrm{~nm}(\mathrm{~B})$. absorbance enhancement and bathochromic displacement caused by TFA suggest that the pyridyl moiety of PyNI becomes protonated when the highly acidic additive is employed. In a non-polar solvent, such as toluene, hydrogenbonded ion pairs $\left(\mathrm{PyNIH}^{+}\right)$are probably formed. It is obvious from the insets in Fig. 1 that the variation in the absorbance at a particular wavelength as a function of the additive concentration exhibits two clearly separated domains. The steep initial rise is attributed to $1: 1$ binding at the pyridyl moiety, whereas the slow absorbance enhancement in the high additive concentration range may imply $1: 2$ complexation. When the initial PyNI concentration is much lower than the total concentration of the additive $\left([\mathrm{H}]_{0}\right)$, the following expression can be derived:

$$
A=A_{0}\left(\frac{1+K_{1}[\mathrm{H}]_{0} \varepsilon_{1}^{\lambda} / \varepsilon_{0}^{\lambda}+K_{1} K_{2}[\mathrm{H}]_{0}^{2} \varepsilon_{2}^{\lambda} / \varepsilon_{0}^{\lambda}}{1+K_{1}[\mathrm{H}]_{0}+K_{1} K_{2}[\mathrm{H}]_{0}^{2}}\right)
$$

where $A_{0}$ is the initial absorbance and $\varepsilon_{0}^{\lambda}, \varepsilon_{1}^{\lambda}$ and $\varepsilon_{2}^{\lambda}$ represent the molar absorption coefficients at a particular wavelength, $\lambda$, for free PyNI and the $1: 1$ and $1: 2$ complexes, respectively. The equilibrium constants for $1: 1\left(K_{1}\right)$ and $1: 2\left(K_{2}\right)$ complex formation are calculated from the non-linear least-squares fit of eqn. 1 to the experimental data. Table 2 gives the averages of the $K_{1}$ and $K_{2}$ values obtained from the fit of the absorbances measured at various wavelengths in the $350-390 \mathrm{~nm}$ range. Both equilibrium constants are markedly larger for TFA than the corresponding values for HFIP, the compound with the weaker hydrogen-bond donor character, but a more pronounced difference appears in the $K_{1}$ values. The binding of TFA to the pyridyl substituent involves proton transfer, leading to a hydrogen-bonded ion pair in which the Coulomb attraction between the ions also enhances the driving force of the complexation. In the $1: 2$ complex, the second additive molecule probably interacts with the oxygen atom of the dicarboximide moiety. As this site is weakly basic, no proton transfer occurs and the stability of the complex is insensitive to the acidity of the hydroxy compound.

Fig. 2(A) reveals that the fluorescence intensity of PyNI grows by a factor of about 11 and the fluorescence peak concomitantly shifts from 21860 to $22120 \mathrm{~cm}^{-1}$ when the TFA concentration is raised to $0.5 \mathrm{mM}$. In contrast, the addition of a greater amount of TFA leads to fluorescence quenching and bathochromic displacement of the spectrum. The inset depicts a plot of the fluorescence quantum yield $\left(\Phi_{\mathrm{F}}\right) v s$. TFA concentration. It can be clearly seen that, after reaching a plateau, $\Phi_{\mathrm{F}}$ diminishes again above a TFA concentration of $2 \mathrm{mM}$. Timeresolved fluorescence measurements proved that dynamic quenching occurs in presence of large amounts of TFA. A plot of the reciprocal fluorescence lifetime $\left(1 / \tau_{\mathrm{F}}\right)$ against TFA concentration is shown in Fig. 3. The linear dependence that emerges is described by the following relationship:

$$
1 / \tau_{\mathrm{F}}=1 / \tau_{\mathrm{F}}^{0}+k_{\mathrm{q}}[\mathrm{TFA}]
$$

where $\tau_{\mathrm{F}}^{0}$ represents the unquenched fluorescence lifetime. From the slope, $k_{\mathrm{q}}=4.3 \times 10^{9} \mathrm{M}^{-1} \mathrm{~s}^{-1}$ is obtained for the quenching rate constant. This value agrees well with that derived from the steady-state measurements $\left(k_{\mathrm{q}}=4.4 \times 10^{9} \mathrm{M}^{-1} \mathrm{~s}^{-1}\right)$. An analogous process is known for the 1-naphtholate anion and has been attributed to the excited-state protonation of the aromatic ring, followed by rapid internal conversion. ${ }^{16}$ TFA was also found to quench photoexcited naphthalene and other aromatic compounds. ${ }^{2 b, 17}$

The fluorescence lifetime of $\mathrm{PyNIH}^{+}$proved to be insensitive to TFA concentration in the $0.3-2 \mathrm{mM}$ range, where PyNI is completely protonated, and the decays were strictly single exponential. At even lower TFA concentrations, dual exponential fluorescence traces were found with constant decay 


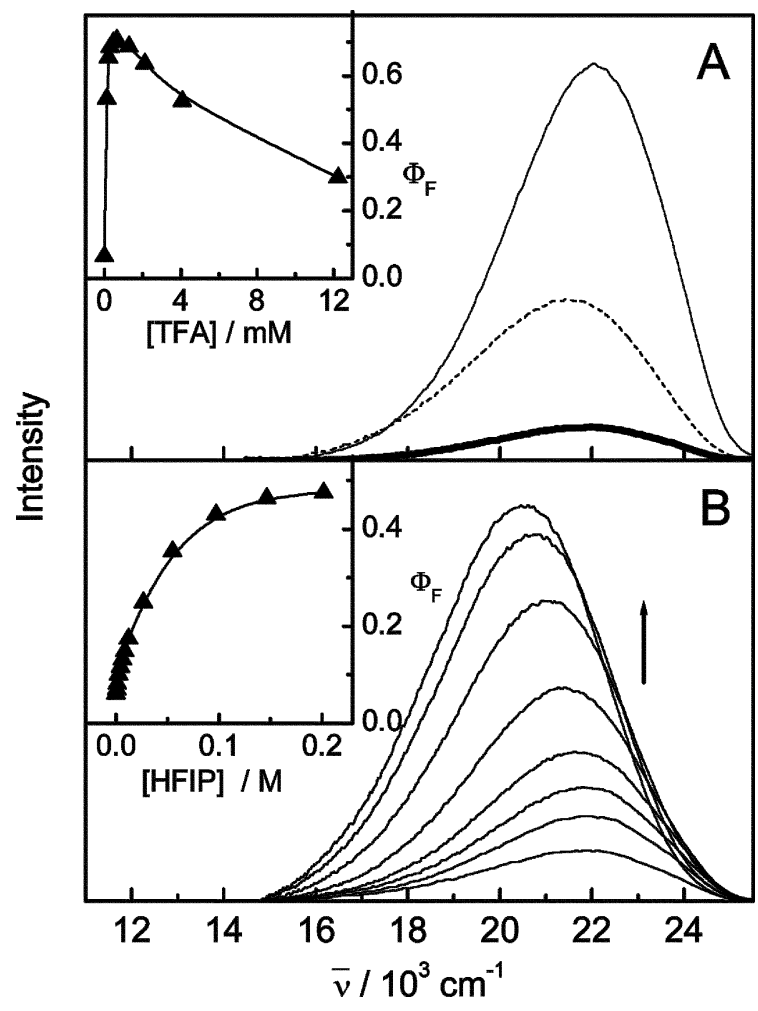

Fig. 2 Change in the fluorescence spectrum of PyNI on addition of TFA (A) and HFIP (B). (A) [TFA] $=0$ (thick line), 0.476 (thin line), 12.3 $\mathrm{mM}$ (dashed line). (B) [HFIP] $=0,0.57,2.5,6.2,12.1,26.4,55.0,97.5$, $146 \mathrm{mM}$. The insets show plots of fluorescence yield as a function of additive concentration. Excitation wavelength $366 \mathrm{~nm}$; $[\mathrm{PyNI}]=0.05$ $\mathrm{mM}$.

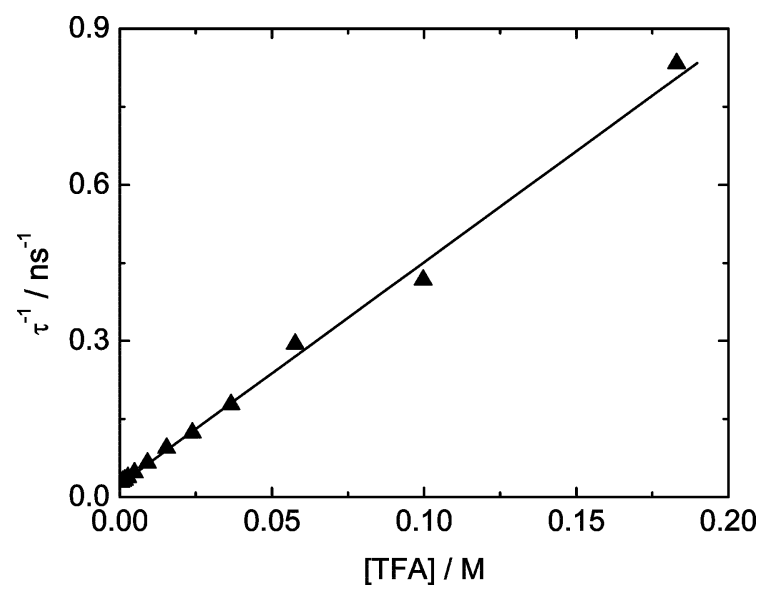

Fig. 3 Variation of the reciprocal fluorescence lifetime of $\mathrm{PyNIH}^{+}$as a function of TFA concentration.

parameters $\tau_{1}=9.7 \mathrm{~ns}$ and $\tau_{2}=33 \mathrm{~ns}$, which correspond to the fluorescence lifetimes of PyNI and $\mathrm{PyNIH}^{+}$, respectively. The relative contribution of the latter component increased with the TFA concentration, indicating that the fraction of $\mathrm{PyNIH}^{+}$ increases at the expense of PyNI.

The addition of increasing amounts of HFIP result in analogous spectral changes to those found in the presence of TFA, but the fluorescence decay kinetics differ markedly. The approximately eightfold enhancement in fluorescence yield, which is accompanied by a $1470 \mathrm{~cm}^{-1}$ red shift of the fluorescence maximum, indicates excited hydrogen-bonded complex formation [Fig. 2(B)]. Representative fluorescence decay curves are displayed in Fig. 4. The time dependence of the fluorescence intensity, $I(t)$, can be well described with a sum of two exponentials:

$$
I(t)=C_{1} \exp \left(-t / \tau_{1}\right)+C_{2} \exp \left(-t / \tau_{2}\right)
$$

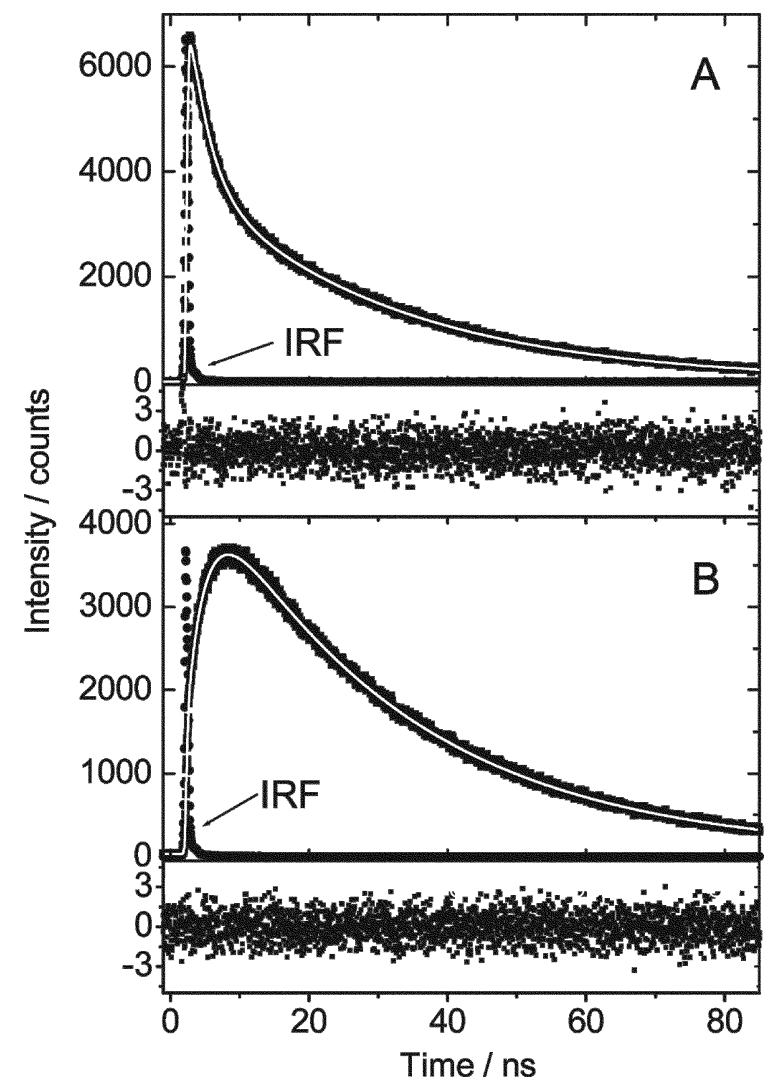

Fig. 4 Fluorescence decay traces recorded at 22730 (A) and 16950 $\mathrm{cm}^{-1}$ (B), instrumental response function (IRF) and the distribution of residuals for PyNI $+0.055 \mathrm{M}$ HFIP in toluene.

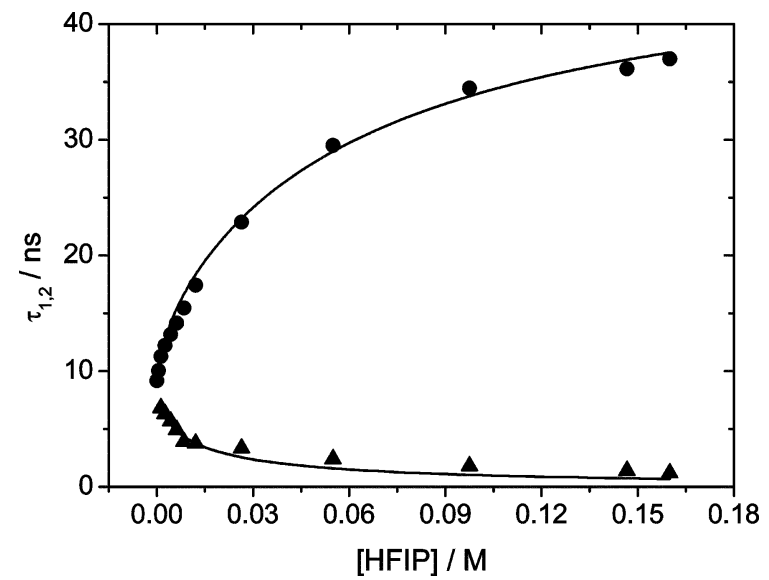

Fig. 5 Plots of fluorescence decay parameters $\tau_{1}(\mathbf{\Delta})$ and $\tau_{2}(\boldsymbol{O}) v s$. HFIP concentration. The lines give the results of the global fitting.

where $\tau_{1}$ and $\tau_{2}$ are the decay parameters and $C_{1}$ and $C_{2}$ denote the amplitudes. The short-lived decay component $\left(\tau_{1}\right)$ at 22730 $\mathrm{cm}^{-1}$ corresponds to the increase in fluorescence at $16950 \mathrm{~cm}^{-1}$, whereas the long-lived components are independent of the detection wavelength. The HFIP concentration dependence of $\tau_{1}$ and $\tau_{2}$ is plotted in Fig. 5. The results suggest reversible complexation both in the ground state and in the excited state. An overview of the processes taken into account in the analysis of the experimental results is presented in Scheme 2. Based on the suggested reaction mechanism, the following relationship can be derived: ${ }^{18}$

$$
\tau_{1,2}=\frac{2}{X+Y \pm \sqrt{(X-Y)^{2}+4 k_{\mathrm{C}} k_{-\mathrm{C}}[\mathrm{H}]}}
$$

where $[\mathrm{H}]$ is the additive concentration, $X=\left(k_{\mathrm{F}}+k_{\mathrm{D}}+k_{\mathrm{C}}[\mathrm{H}]\right)$ and $Y=\left(k_{\mathrm{F}}^{\prime}+k_{\mathrm{D}}^{\prime}+k_{-\mathrm{C}}\right), k_{\mathrm{C}}$ and $k_{-\mathrm{C}}$ are the rate constants 


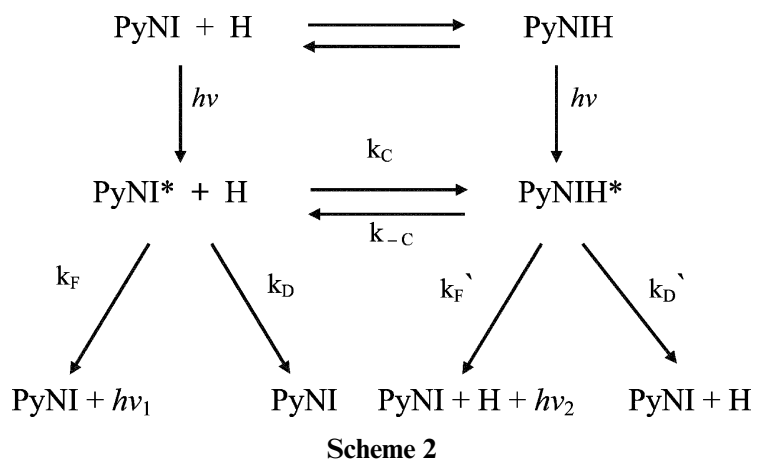

of formation and dissociation of the excited hydrogenbonded complex, respectively, and $k_{\mathrm{F}}$ and $k_{\mathrm{D}}$ represent the rate constants of fluorescence and non-radiative deactivation, respectively, for PyNI. The primed quantities refer to the corresponding processes for the hydrogen-bonded complex. The global fit of eqn. 4 to the experimental data provides the individual rate constants and the lines in Fig. 5 indicate the calculated functions. The results summarised in Table 1 demonstrate that the formation of the excited hydrogen-bonded complex via interaction of excited PyNI with ground-state HFIP is practically a diffusion-controlled process and the slow deactivation rate of the excited complex $\left(k_{\mathrm{F}}{ }^{\prime}+k_{\mathrm{D}}{ }^{\prime}=2.1 \times 10^{7}\right.$ $\mathrm{s}^{-1}$ ) permits the interconversion between free and bound PyNI in the excited state. The equilibrium constant of the hydrogen bonding in the excited state $\left(K_{\text {exc }}=k_{\mathrm{C}} / k_{-\mathrm{C}}=80 \mathrm{M}^{-1}\right)$ is somewhat smaller than that found in the ground state $\left(K=130 \mathrm{M}^{-1}\right)$. The difference is presumably related to the lower electron density on the heterocyclic nitrogen of the pyridyl moiety in the excited state.

\section{Photophysical properties}

It is interesting to compare the photophysical properties of PyNI to those of its protonated and hydrogen-bonded form The investigations have been carried out in the presence of $0.16 \mathrm{M}$ HFIP or $0.47 \mathrm{mM}$ TFA. These additive concentrations ensure complete $1: 1$ complexation. Because no photochemical reaction occurs, the quantum yield of internal conversion $\left(\Phi_{\mathrm{IC}}\right)$ is derived using the measured fluorescence $\left(\Phi_{\mathrm{F}}\right)$ and triplet yields $\left(\Phi_{\text {ISC }}\right)$ :

$$
\Phi_{\mathrm{IC}}=1-\Phi_{\mathrm{F}}-\Phi_{\mathrm{ISC}}
$$

The most remarkable feature of the results presented in Table 2 is the marked fluorescence yield enhancement upon both protonation and hydrogen bonding. The particularly high $\Phi_{\mathrm{F}}$ of $\mathrm{PyNI}^{+}$may permit its utilisation in fluorescent probes and fluorescent labelling methods. The binding of the hydroxy compounds to PyNI slows down the fluorescence decay and reduces $\Phi_{\text {IC }}$ as well as $\Phi_{\text {ISC }}$.

To gain a deeper insight into the competition among the energy dissipation processes, the rate constants for fluorescence emission $\left(k_{\mathrm{F}}\right)$, intersystem crossing $\left(k_{\mathrm{ISC}}\right)$ and internal conversion $\left(k_{\mathrm{IC}}\right)$ were calculated using the expressions below:

$$
\begin{gathered}
k_{\mathrm{ISC}}=\Phi_{\mathrm{ISC}} / \tau_{\mathrm{F}} \\
k_{\mathrm{IC}}=\left(1-\Phi_{\mathrm{ISC}}-\Phi_{\mathrm{F}}\right) / \tau_{\mathrm{F}} \\
k_{\mathrm{F}}=\Phi_{\mathrm{F}} / \tau_{\mathrm{F}}
\end{gathered}
$$

It is evident from Table 2 that the considerable increase in fluorescence upon complexation is due primarily to the deceleration of the radiationless processes; the increase in $k_{\mathrm{F}}$ makes less of a contribution. The radiative rate constant increases in the order PyNI $<$ PyNIH $<\mathrm{PyNIH}^{+}$, whereas the rate con-
Table 1 Kinetic parameters of PyNI in the presence of HFIP in toluene

$\begin{array}{ll}k_{\mathrm{F}}+k_{\mathrm{D}} / 10^{7} \mathrm{~s}^{-1} & 10.3 \\ k_{\mathrm{C}} / 10^{7} \mathrm{M}^{-1} \mathrm{~s}^{-1} & 800 \\ k_{-\mathrm{C}} / 10^{7} \mathrm{~s}^{-1} & 10 \\ k_{\mathrm{F}}^{\prime}+k_{\mathrm{D}}^{\prime} / 10^{7} \mathrm{~s}^{-1} & 2.1 \\ k_{\mathrm{C}} / k_{-\mathrm{C}} / \mathrm{M}^{-1} & 80\end{array}$

Table 2 Photophysical parameters of PyNI solutions in deoxygenated toluene

\begin{tabular}{llll}
\hline & PyNI $^{a}$ & PYNI $+\mathrm{HFIP}^{b}$ & ${\text { PyNI }+\mathrm{TFA}^{c}}^{c}$ \\
\hline$K_{1} / \mathrm{M}^{-1}$ & & 130 & 2400 \\
$K_{2} / \mathrm{M}^{-1}$ & & 1.9 & 3 \\
$\bar{v}_{\text {max }}($ absorption $) / \mathrm{cm}^{-1}$ & 28900 & 28600 & 28370 \\
$v_{\max }($ fluorescence $) / \mathrm{cm}^{-1}$ & 21860 & 20390 & 22100 \\
$E\left(\mathrm{~S}_{1} / \mathrm{eV}\right.$ & 3.066 & 3.014 & 3.045 \\
$\tau_{\mathrm{F}} / \mathrm{ns}$ & 9.7 & 37 & 33 \\
$\Phi_{\mathrm{F}}$ & 0.061 & 0.50 & 0.70 \\
$\Phi_{\mathrm{ISC}}$ & 0.14 & 0.085 & 0.059 \\
$\Phi_{\mathrm{IC}}$ & 0.80 & 0.41 & 0.24 \\
$k_{\mathrm{F}} / 10^{7} \mathrm{~s}^{-1}$ & 0.63 & 1.4 & 2.1 \\
$k_{\mathrm{ISC}} / 10^{7} \mathrm{~s}^{-1}$ & 1.4 & 0.23 & 0.18 \\
$k_{\mathrm{IC}} / 10^{7} \mathrm{~s}^{-1}$ & 8.2 & 1.1 & 0.73
\end{tabular}

${ }^{a}$ Data from ref. 12. ${ }^{b}$ All parameters except the equilibrium constants were measured in the presence of $0.16 \mathrm{M}$ HFIP. ${ }^{c}$ All parameters except the equilibrium constants were measured in the presence of $0.47 \mathrm{mM}$ TFA

stants of intersystem crossing and internal conversion exhibit opposite trends.

The interpretation of the results can be based on the knowledge obtained from previous studies of PyNI ${ }^{12}$ and $N$ phenyl-1,2-naphthalimide (PhNI). ${ }^{19,20}$ It has been demonstrated that the energy gap between the close-lying $\mathrm{S}_{1}\left(\pi \pi^{*}\right)$ and $\mathrm{S}_{2}\left(\mathrm{n} \pi^{*}\right)$ excited states controls the rate of radiationless deactivation and the extended conjugation, including both the phenyl and the naphthalimide moieties, stabilises the $\mathrm{S}_{2}$ state. The introduction of ortho-methyl or the para- $\mathrm{CF}_{3}$ substituents on PhNI has been found to hinder the conjugation of the two aromatic systems within the molecule and, thereby, to increase the $S_{2}$ state energy. The hydrogen bonding and, particularly, the protonation of PyNI have a similar effect: they hamper the delocalisation of the lone-pair electrons of the heterocyclic nitrogen of the pyridyl ring and, as a result of that, expand the energy gap between the $S_{1}$ and $S_{2}$ singlet excited states. The enlarged energy difference permits less effective vibrational coupling between the adjacent states and, therefore, the internal conversion slows down significantly. The lower $k_{\mathrm{IC}}$ for $\mathrm{PyNIH}^{+}$compared with that of PyNIH probably reflects the larger $\mathrm{S}_{1}-\mathrm{S}_{2}$ energy separation in the former. Table 2 clearly shows that the rate constant of intersystem crossing changes parallel with $k_{\mathrm{IC}}$. It is well established that both the $S_{1}$ and the lowest triplet $\left(T_{1}\right)$ states of 1,2-naphthalimides possess $\pi \pi^{*}$ character $^{10}$ and the transition between $\pi \pi^{*}$ states is unfavourable according to the El-Sayed rule. $^{21}$ This explains the fairly slow intersystem crossing for PyNIH and PyNIH ${ }^{+}$. The larger $k_{\text {ISC }}$ of PyNI may imply the involvement of $n \pi^{*}$ states of higher energy in the triplet formation.

\section{Exciplex formation with naphthalene}

The addition of naphthalene brings about marked changes in the fluorescence spectra (Fig. 6) but no interaction can be detected in the ground state. In the case of PyNIH fluorescence, quenching merely occurs, but the emergence of a new band can also be observed for PyNI and $\mathrm{PyNIH}^{+}$. The insets show representative examples of the resolution of the fluorescence spectra. The fluorescence maxima are compiled in Table 3 . The band located at low energy is assigned to exciplex fluorescence. 


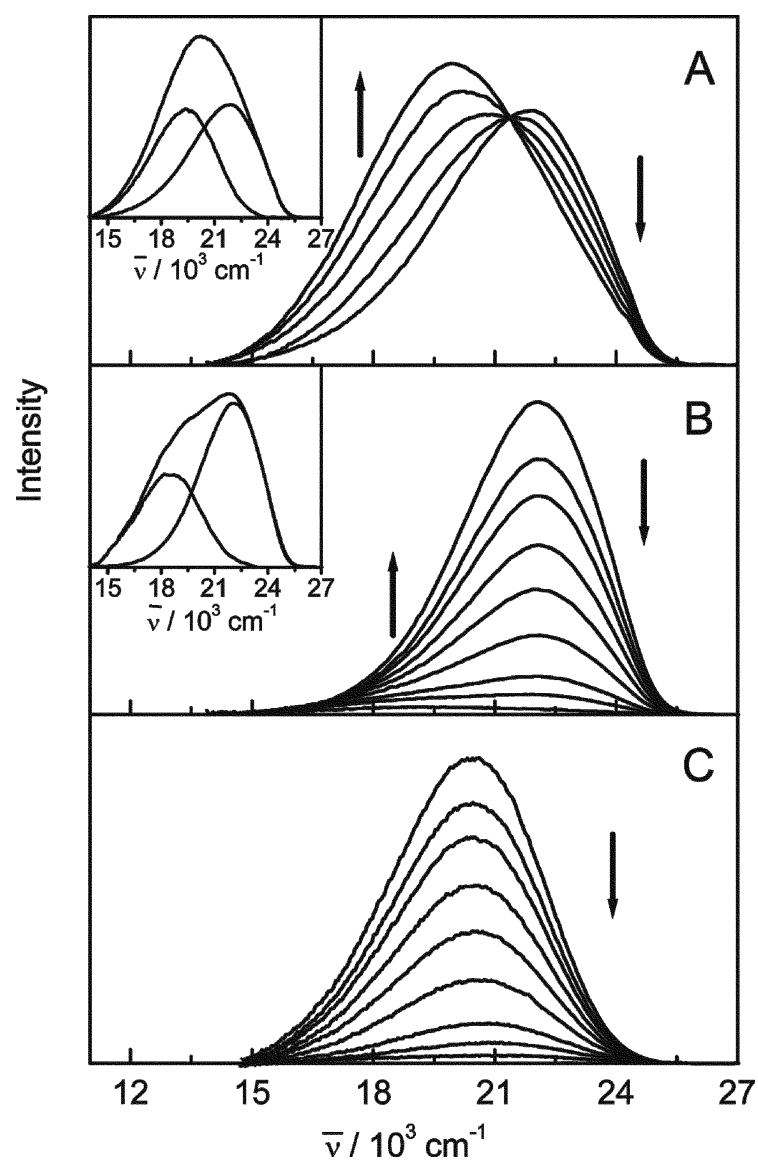

Fig. 6 (A) Change in the fluorescence spectrum of PyNI on addition of $0,0.038,0.10,0.183,0.267 \mathrm{M}$ naphthalene. (B) Fluorescence spectrum of $\mathrm{PyNIH}^{+}$in the presence of $0,0.8,1.6,3.2,6.0,11.8,29.1$, $60.0 \mathrm{mM}$ naphthalene. (C) Fluorescence quenching of PyNIH by $0,0.8$, $1.6,3.2,6.0,12,29,60,167 \mathrm{mM}$ naphthalene. The insets shows the resolution of the fluorescence band: [naphthalene] $=0.18$ (A) and 0.06 M (B). Excitation wavelength $366 \mathrm{~nm}$; absorbance 0.1.

Table 3 Spectroscopic and kinetic parameters of exciplexes with naphthalene in toluene

\begin{tabular}{|c|c|c|}
\hline & PyNI & $\mathrm{PyNIH}^{+}$ \\
\hline $\bar{v}_{\max }($ fluorescence $) / \mathrm{cm}^{-1}$ & 21860 & 22100 \\
\hline$v_{\max }($ exciplex fluorescence $) / \mathrm{cm}^{-1}$ & 19450 & 18540 \\
\hline$\Delta \bar{v}_{\max } / \mathrm{cm}^{-1}$ & 2410 & 3560 \\
\hline$k_{\mathrm{C}}^{\mathrm{exc}} / 10^{7} \mathrm{M}^{-1} \mathrm{~s}^{-1}$ & 370 & 750 \\
\hline$k_{-\mathrm{C}}^{\mathrm{exc}} / 10^{7} \mathrm{~s}^{-1}$ & 57 & 2 \\
\hline$K_{\mathrm{exc}} / \mathrm{M}^{-1}$ & 6.5 & 375 \\
\hline$\Delta G_{\text {exc }} / \mathrm{eV}$ & -0.048 & -0.15 \\
\hline$k_{\mathrm{F}} / 10^{7} \mathrm{~s}^{-1}$ & 0.63 & 2.1 \\
\hline$k_{\mathrm{D}} / 10^{7} \mathrm{~s}^{-1}$ & 9.6 & 0.91 \\
\hline$k_{\mathrm{F}}^{\mathrm{exc}}+k_{\mathrm{D}}^{\mathrm{exc}} / 10^{7} \mathrm{~s}^{-1}$ & 4.0 & 16 \\
\hline$k_{\mathrm{F}}^{\mathrm{exc}} / 10^{7} \mathrm{~s}^{-1}$ & 0.5 & 0.4 \\
\hline$k_{\mathrm{D}}^{\mathrm{exc}} / 10^{7} \mathrm{~s}^{-1}$ & 3.5 & 15.6 \\
\hline
\end{tabular}

Samanta and Saroja have also found exciplex formation in the reaction of the excited 1,8-naphthalimide with naphthalene. ${ }^{8}$ The fluorescence of the $\mathrm{PyNIH}^{+}$exciplex is centred at lower energy than that of the PyNI exciplex, implying a larger driving force for exciplex formation $(\Delta G)$ in the former case.

Fig. 7 presents the variation of the fluorescence yields $\left(\Phi_{\mathrm{F}}\right)$ with increasing naphthalene concentration. In contrast with the other systems, the fluorescence yields of neither PyNI nor its exciplex level off, even in the presence of high amounts of naphthalene, which indicates slower quenching. The steep initial change in $\Phi_{\mathrm{F}}$ for both $\mathrm{PyNIH}^{+}$and its exciplex suggests close to diffusion-controlled exciplex formation. The $\Phi_{\mathrm{F}}$ of PyNIH varies parallel with that of $\mathrm{PyNIH}^{+}$, but for the former species, the low intensity of the exciplex emission precludes

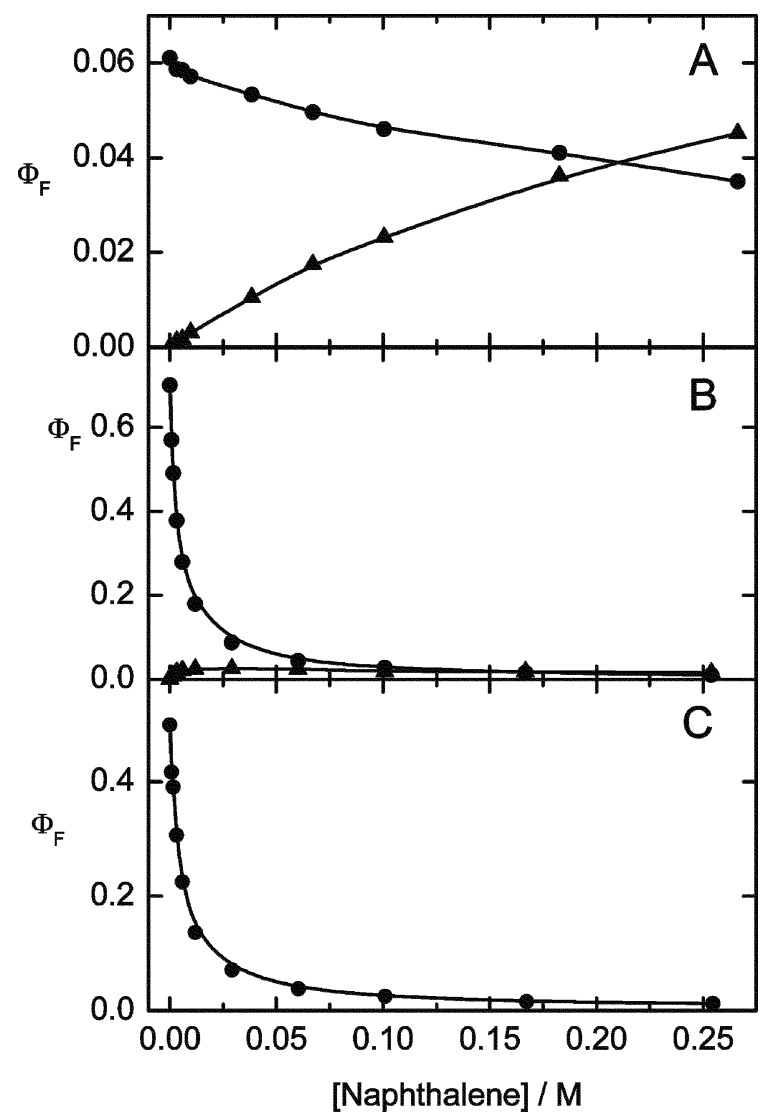

Fig. 7 Fluorescence yields of (A) PyNI, (B) $\mathrm{PyNIH}^{+}$and (C) PyNIH (O) and their respective exciplexes $(\boldsymbol{A})$ as a function of naphthalene concentration.

detection of the exciplex band. Thus, time-resolved measurements have been performed only with PyNI and $\mathrm{PyNIH}^{+}$.

The emission of these species, monitored at $22220 \mathrm{~cm}^{-1}$, showed double-exponential decay kinetics (eqn. 3), whose fast component matched the build-up of the exciplex fluorescence at $15870 \mathrm{~cm}^{-1}$. The decay time of the long-lived component agreed well throughout the spectra. These observations obviously imply reversible exciplex formation. In Scheme 3, P*

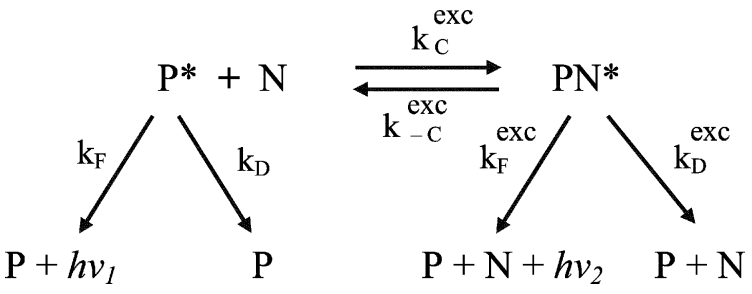

Scheme 3

denotes the excited naphthalimide derivative and $\mathrm{N}$ is naphthalene. The fluorescence decay parameters, obtained from the fit of eqn. 3 to the fluorescence traces, are plotted as a function of the naphthalene concentration in Fig. 8. The $\tau_{1}$ values exhibit similar changes for both systems, but opposite trends are discernible for the $\tau_{2}$ parameters. In the case of PyNI, slow growth is found, while after the steep decrease, $\tau_{2}$ tends to level off for $\mathrm{PyNIH}^{+}$. To elucidate all of the rate constants pertaining to Scheme 3, global analysis of the naphthalene concentration dependence of $\tau_{1}$ and $\tau_{2}$ has been performed. The lines in Fig. 8 give the best fit of the experimental data and the calculated kinetic parameters are summarised in Table 3. In order to obtain $k_{\mathrm{F}}^{\mathrm{exc}}$ and $k_{\mathrm{D}}^{\mathrm{exc}}$, the ratio of the fluorescence quantum yields for the exciplex and the naphthalimide, $\Phi_{\mathrm{F}}(\mathrm{PN}) / \Phi_{\mathrm{F}}(\mathrm{P})$, are plotted as a function of naphthalene concentration (Fig. 9). A linear dependence is observed, which can be rationalised by the following expression: 


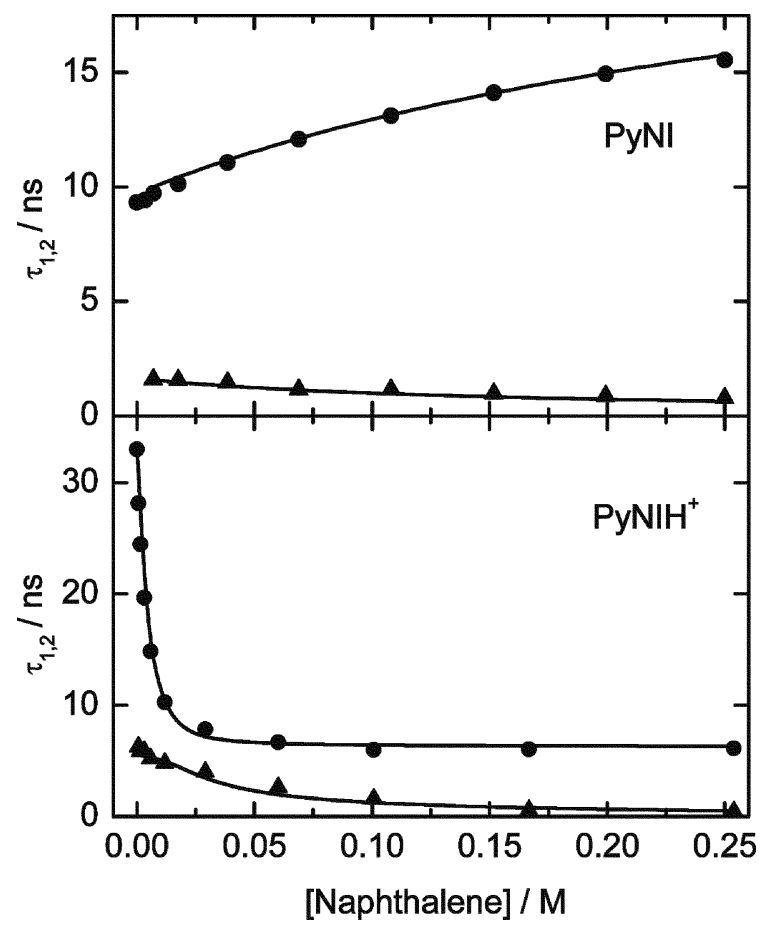

Fig. 8 Naphthalene concentration dependence of the fluorescence decay parameters $\tau_{1}(\boldsymbol{\Delta})$ and $\tau_{2}(\boldsymbol{O})$ for PyNI and $\mathrm{PyNIH}^{+}$.

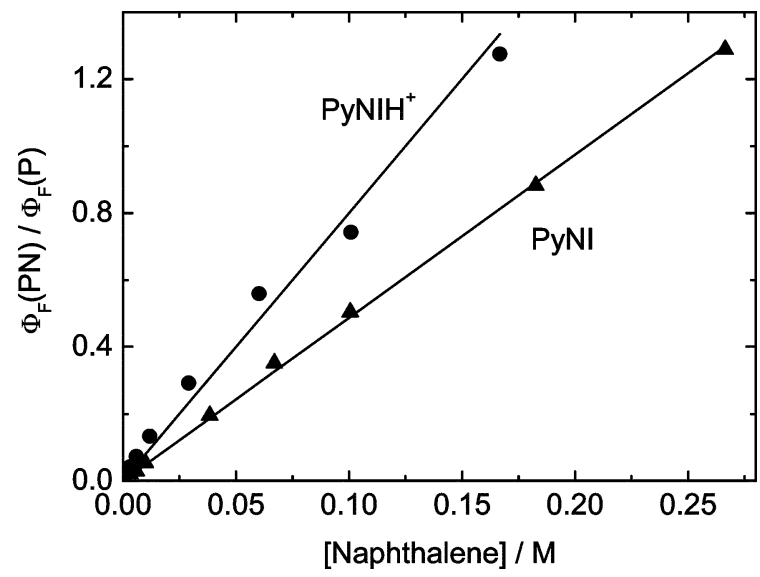

Fig. 9 Exciplex and naphthalimide [PyNI (A), $\mathrm{PyNIH}^{+}$ fluorescence yield ratios plotted against naphthalene concentration.

$$
\frac{\Phi_{\mathrm{F}}(\mathrm{PN})}{\Phi_{\mathrm{F}}(\mathrm{P})}=\frac{k_{\mathrm{F}}^{\mathrm{exc}} k_{\mathrm{C}}^{\mathrm{exc}}[\mathrm{N}]}{k_{\mathrm{F}}\left(k_{\mathrm{F}}^{\mathrm{exc}}+k_{\mathrm{D}}^{\mathrm{exc}}+k_{-\mathrm{C}}^{\mathrm{exc}}\right)}
$$

where the designations are defined in Scheme 3. From the slopes, values of $k_{\mathrm{F}}^{\mathrm{exc}}=5 \times 10^{6}$ and $4 \times 10^{6} \mathrm{~s}^{-1}$ are calculated for the exciplexes of PyNI and $\mathrm{PyNIH}^{+}$, respectively, using the kinetic parameters determined from the time-resolved measurements.

In Table 3, the most appreciable difference is seen in the rate constant of exciplex dissociation $\left(k_{-C}^{\mathrm{exc}}\right)$. The exciplex formation is diffusion controlled for $\mathrm{PyNIH}^{+}$, while it falls below this limit by a factor of $c a .2$ for PyNI. The equilibrium constant and the change in free energy for exciplex formation are derived as $K_{\text {exc }}=k_{\mathrm{C}}^{\mathrm{exc}} / k_{-\mathrm{C}}^{\mathrm{exc}}$ and $\Delta G_{\mathrm{exc}}=-R T \ln \left(k_{\mathrm{C}}^{\mathrm{exc}} / k_{-\mathrm{C}}^{\mathrm{exc}}\right)$, respectively. In accordance with the more substantial red shift of the fluorescence spectrum (vide supra), we have found that exciplex formation is more exergonic for $\mathrm{PyNIH}^{+}$than for PyNI. This fact results in low $k_{-\mathrm{C}}^{\text {exc }}$ and diffusion-controlled exciplex production in the former case. Cyclic voltammetric measurements in benzonitrile also demonstrate that $\mathrm{PyNIH}^{+}$is a better electron acceptor. The reversible one-electron reduction wave of PyNI at $E_{\text {red }}=-1.60 \mathrm{~V} v s$. ferrocene became irreversible on addition of
TFA, and the peak appeared at about $300 \mathrm{mV}$ less negative potential. The lower energy of the $\mathrm{PyNIH}^{+}$exciplex permits more efficient vibronic coupling to the ground state, which in turn accelerates the radiationless deactivation. This seems to be the reason for the higher $k_{\mathrm{D}}^{\text {exc }}$ value of the $\mathrm{PyNIH}^{+}$exciplex. The low fluorescence yield of this exciplex arises mainly from the rapid energy dissipation.

\section{Conclusions}

The addition of strong hydrogen-bond donors, such as trifluoroacetic acid and hexafluoro-2-propanol, to solutions of $N$-(4-pyridyl)-1,2-naphthalimide in toluene exerts a profound effect on the kinetics of the excited-state depopulation pathways and the exciplex formation with naphthalene. Even a trace amount of TFA causes considerable fluorescence enhancement and the fluorescence yield reaches values as high as 0.70 . This phenomenon is mainly due to the deceleration of the internal conversion, which in turn arises from the expanded energy gap between the $S_{1}$ and $S_{2}$ singlet excited states of the TFA-PyNI complex. Analogous photophysical changes have also been observed in the presence of HFIP, but the equilibrium constants of HFIP binding to PyNI are significantly lower, both in the excited state and in the ground state, than those of the TFA complex. Fluorescence quenching of PyNI and its TFA complex by naphthalene proceeds via reversible exciplex formation. In the latter case, the energy of the exciplex is reduced because the complexation of PyNI by TFA makes the reduction potential less negative. The results of this study may be applied to the design of fluorescent probes and organic light-emitting diodes.

\section{Acknowledgements}

The authors very much appreciate the support of this work by the Hungarian Scientific Research Fund (OTKA, Grant T034990) and NKFP Medichem Project 1/047.

\section{References}

1 (a) K. Ohkura and K. Seki, Photoinduced transannular reaction of 1,3-dimethylcyclooctapyrimidine-2,4-diones, Photochem. Photobiol., 2002, 75, 579-584; (b) K. Ohkura, K. Nishijima and K. Seki, Electrocyclic rearrangement of pentamethylcyclooctapyrimidine2,4-diones: reaction pathway into a 9,11-diazapentacyclo$\left[6.4 .0 .0^{1,3} \cdot 0^{2,5} \cdot 0^{4,8}\right]$ dodecane system, and a 9,11-diazapentacyclo $\left[6.4 .0 .0^{1,3} \cdot 0^{2,6} .0^{4,8}\right]$ dodecane system, Photochem. Photobiol., 2002, 74, 385-390; (c) K. Ohkura, K. Matsuda and K. Seki, Acid-catalyzed photoreaction of 5-chloro-1,3-dimethyluracil with substituted benzenes, Heterocycles, 1991, 32, 1371-1376; (d) N. J. Bruce, Y. Kumar, I. Ravanal and S. Safe, Photochemistry of chlorinated biphenyls in iso-octane solution, J. Chem. Soc., Perkin Trans. 2, 1978, 880-884.

2 (a) N. J. Bunce and M. D. Bergsma, Trifluoroacetic-acid quenching of naphthalene fluorescence - implications for the mechanism of photoelectrophilic hydrogen-exchange, J. Org. Chem., 1980, 45, 2083-2086; (b) N. J. Bunce, Y. Kumar and L. Ravanal, Interaction of photoexcited naphthalenes with trifluoroacetic acid, J. Org. Chem., 1979, 44, 2612-2616.

3 (a) D. Gust, T. A. Moore, A. L. Moore, X. C. C. Ma, R. A. Nieman, G. R. Seely, R. E. Belford and J. E. Lewis, Proton-assisted photoinduced electron-transfer from porphyrin to quinone - a photo-CIDNP and laser flash-photolysis study, J. Phys. Chem., 1991, 95, 4442-4445; (b) L. Biczók and H. Linschitz, Oxidation of triplet $\mathrm{C}_{60}$ by hydrogen-bonded chloranil: efficient formation, spectrum and charge-shift reactions of $\mathrm{C}_{60}{ }^{+}$cation radical, J. Phys. Chem. A, 2001, 105, 11051-11056.

4 E. C. A. Ojadi, H. Linschitz, M. Gouterman, R. I. Walter, J. S. Lindsey, R. W. Wagner, P. R. Droupadi and W. D. Wang, Sequential protonation of meso-(p-(dimethylamino)phenyl)porphyrins. Charge-transfer excited-states producing hyperporphyrins, J. Phys. Chem., 1993, 97, 13 192-13 197.

5 (a) F. Cosnard and V. Wintgens, A new fluoroionophore derived from 4-amino-N-methyl-1,8-naphthalimide, Tetrahedron Lett., 1998, 
39, 2751-2754; (b) D. P. Adhikiri and M. D. Heagy, Fluorescent chemosensor for carbohydrates which shows large change in chelation-enhanced quenching, Tetrahedron Lett., 1999, 40, 7893 7896; (c) H. Cao, D. I. Diaz, N. DiCesare, J. R. Lakowicz and M. D. Heagy, Monoboronic acid sensor that displays anomalous fluorescence sensitivity to glucose, Org. Lett., 2002, 4, 1503-1505; (d) B. Ramachandram, N. B. Sankaran, R. Karmakar, S. Saha and A. Samanta, Fluorescence signalling of transition metal ions by multi-component systems comprising 4-chloro-1,8-naphthalimide as fluorophore, Tetrahedron, 2000, 56, 7041-7044; (e) I. Grabchev, X. H. Qian, Y. Xiao and R. Zhang, Novel heterogeneous PET fluorescent sensors selective for transition metal ions or protons: polymers regularly labelled with naphthalimide, New J. Chem., 2002 , 26, 920-925; $(f)$ I. Grabchev, J. M. Chovelon and X. Qian, A copolymer of 4-N,N-dimethylaminoethylene-N-allyl-1,8naphthalimide with methylmethacrylate as a selective fluorescent chemosensor in homogeneous systems for metal cations, J. Photochem. Photobiol., A., 2003, 158, 37-43.

6 (a) H. Tian, J. A. Gan, K. C. Chen, J. He, Q. L. Song and X. Y. Hou, Positive and negative fluorescent imaging induced by naphthalimide polymers, J. Mater. Chem., 2002, 12, 1262-1267; (b) J. A. Gan, H. Tian and K. C. Chen, Photoacid-induced fluorescent imaging by copolymer containing naphthalimide, Polym. Adv. Technol., 2002, 13, 584-588.

7 D. Kolosov, V. Adamovich, P. Djurovich, M. E. Thompson and C. Adachi, 1,8-naphthalimides in phosphorescent organic LEDs: the interplay between dopant, exciplex, and host emission, J. Am. Chem. Soc., 2002, 124, 9945-9954.

8 A. Samanta and G. Saroja, Steady-state and time-resolved studies on the redox behavior of 1,8-naphthalimide in the excited-state, J. Photochem. Photobiol., A, 1994, 84, 19-26.

9 Y. Kubo, M. Suto, S. Tojo and T. Araki, Photochemical reaction of $\mathrm{N}$-methylnaphthalene-1,8-dicarboximide with alkenes and furans. Cyclobutane and oxetane formation, J. Chem. Soc., Perkin Trans. 1 , 1986, 771-779.

10 V. Wintgens, P. Valat, J. Kossanyi, L. Biczók, A. Demeter and T. Bérces, Spectroscopic properties of aromatic dicarboximides. Part. 2. Substituent effect on the photophysical properties of
N-phenyl-1,2-naphthalimide, J. Chem. Soc., Faraday Trans., 1994, 90, 411-421.

11 Y. Kubo, S. Tojo, M. Suto, R. Toda and T. Araki, Photochemical reactions of naphthalenedicarboximides. Effect of arene structure in imide compounds on reaction with olefins, Chem. Lett., 1984, 2075-2078

$12 \mathrm{~K}$. Nagy and L. Biczók, Effect of N-pyridyl substitution and hydrogen bonding on the deactivation of singlet excited 1,2naphthalimide, Res. Chem. Intermed., 2002, 28, 837-847.

13 D. F. Eaton, Reference materials for fluorescence measurement, Pure Appl. Chem., 1988, 60, 1107-1114.

14 L. Biczók, T. Bérces and H. Inoue, Effects of molecular structure and hydrogen-bonding on the radiationless deactivation of singlet excited fluorenone derivatives, J. Phys. Chem. A, 1999, 103, 3837-3842.

15 M. H. Abraham, P. P. Duce, P. P. Prior, D. G. Barratt, J. J. Morris and P. J. Taylor, Hydrogen Bonding. Part 9. Solute proton donor and proton acceptor scales for use in drug design, J. Chem. Soc., Perkin Trans. 2, 1989, 1355-1375.

16 L. M. Tolbert and K. M. Solntsev, Excited-state proton transfer: From constrained systems to "super" photoacids to superfast proton transfer, Acc. Chem. Res., 2002, 35, 19-27.

17 N. J. Bunce and Y. Kumar, Interaction of photoexcited aromatic compounds with trifluoroacetic acid, J. Photochem., 1978, 9, 287-289.

18 J. B. Birks, Photophysics of Aromatic Molecules, Wiley-Interscience, London, 1970, p. 304.

19 A. Demeter, T. Bérces, L. Biczók, V. Wintgens, P. Valat and J. Kossanyi, Comprehensive model of the photophysics of N-phenyl-naphthalimides: the role of solvent- and rotational relaxation, J. Phys. Chem., 1996, 100, 2001-2011.

20 A. Demeter, T. Bérces, L. Biczók, V. Wintgens, P. Valat and J. Kossanyi, Spectroscopic properties of aromatic dicarboximides. Part. 2. Substituent effect on the photophysical properties of N-phenyl-1,2-naphthalimide, J. Chem. Soc., Faraday Trans., 1994, 90, 2635-2641.

21 M. A. El-Sayed, Spin-orbit coupling and the radiationless processes in nitrogen heterocyclics, J. Chem. Phys., 1963, 38, 2834-2838. 